\title{
Cognitive function and risks of cardiovascular disease and hypoglycaemia in patients with type 2 diabetes: the Action in Diabetes and Vascular Disease: Preterax and Diamicron Modified Release Controlled Evaluation (ADVANCE) trial
}

\author{
B. E. de Galan $\cdot$ S. Zoungas $\cdot$ J. Chalmers . \\ C. Anderson • C. Dufouil • A. Pillai • M. Cooper • \\ D. E. Grobbee $\cdot$ M. Hackett $\cdot$ P. Hamet $\cdot$ S. R. Heller • \\ L. Lisheng $\cdot$ S. MacMahon $\cdot$ G. Mancia $\cdot$ B. Neal $\cdot$ \\ C. Y. Pan - A. Patel • N. Poulter • F. Travert • \\ M. Woodward · for the ADVANCE Collaborative group
}

Received: 29 May 2009 / Accepted: 13 July 2009 / Published online: 18 August 2009

(C) Springer-Verlag 2009

\begin{abstract}
Aims/hypothesis The relationship between cognitive function, cardiovascular disease and premature death is not well established in patients with type 2 diabetes. We assessed the effects of cognitive function in 11,140 patients with type 2 diabetes who participated in the Action in Diabetes and Vascular
\end{abstract}

B. E. de Galan $\cdot$ S. Zoungas $(\bowtie) \cdot J$. Chalmers $\cdot$ C. Anderson • A. Pillai $\cdot$ M. Hackett $\cdot$ S. MacMahon $\cdot$ B. Neal $\cdot$ A. Patel $\cdot$ M. Woodward

The George Institute for International Health,

The University of Sydney, PO Box M201,

Missenden Road, Camperdown,

Sydney, NSW 2050, Australia

e-mail: szoungas@george.org.au

B. E. de Galan

Radboud University Nijmegen Medical Centre,

Nijmegen, the Netherlands

S. Zoungas

Monash University, Melbourne, VIC, Australia

C. Dufouil

INSERM U708, Paris, France

C. Dufouil $\cdot$ F. Travert

UPMC University of Paris, Paris, France

\section{Cooper}

Baker IDI Heart Research Institute,

Melbourne, VIC, Australia

\section{E. Grobbee}

Julius Centre for Health Sciences and Primary Care,

University Medical Centre Utrecht, Utrecht, the Netherlands
Disease: Preterax and Diamicron Modified Release Controlled Evaluation (ADVANCE) trial. Furthermore, we tested whether level of cognitive function altered the beneficial effects of the BP-lowering and glycaemic-control regimens in the trial. Methods Cognitive function was assessed using the Mini Mental State Examination at baseline, and defined by

P. Hamet

Centre Hospitalier de l'Université de Montreal and Université de Montreal,

Montreal, QC, Canada

\section{S. R. Heller}

University of Sheffield and Sheffield Teaching Hospitals National Health Service Foundation Trust,

Sheffield, UK

L. Lisheng

Chinese Hypertension League Institute,

Beijing, China

G. Mancia

University of Milan-Bicocca and San Gerardo Hospital,

Milan, Italy

C. Y. Pan

Chinese People's Liberation Army General Hospital,

Beijing, China

N. Poulter

Imperial College and St Mary's Hospital,

London, UK

M. Woodward

Mount Sinai School of Medicine,

New York, NY, USA 
scores 28-30 ('normal', $n=8,689$ ), 24-27 ('mild dysfunction', $n=2,231$ ) and $<24$ ('severe dysfunction', $n=212$ ). Risks of major cardiovascular events, death and hypoglycaemia and interactions with treatment were assessed using Cox proportional hazards analysis.

Results Relative to normal function, both mild and severe cognitive dysfunction significantly increased the multipleadjusted risks of major cardiovascular events (HR 1.27, 95\% CI 1.11-1.46 and 1.42, 95\% CI 1.01-1.99; both $p<$ $0.05)$, cardiovascular death $(1.41,95 \%$ CI $1.16-1.71$ and $1.56,95 \%$ CI $0.99-2.46$; both $p \leq 0.05)$ and all-cause death (1.33, 95\% CI 1.16-1.54 and 1.50, 95\% CI 1.06-2.12; both $p<0.03)$. Severe, but not mild, cognitive dysfunction increased the risk of severe hypoglycaemia (HR 2.10, 95\% CI 1.14-3.87; $p=0.018)$. There was no evidence of heterogeneity of treatment effects on cardiovascular outcomes in subgroups defined by cognitive function at baseline.

Conclusions/interpretation Cognitive dysfunction is an independent predictor of clinical outcomes in patients with type 2 diabetes, but does not modify the effects of BP lowering or glucose control on the risks of major cardiovascular events.

Trial registration: ClinicalTrials.gov NCT00145925

Funding: Supported by grants from Servier and from the National Health and Medical Research Council of Australia.

Keywords Blood pressure control - Cardiovascular disease risk · Cognitive function · Glucose control · Hypoglycaemia · MMSE $\cdot$ Mortality $\cdot$ Type 2 diabetes

$\begin{array}{ll}\text { Abbreviations } \\ \text { ADVANCE } & \begin{array}{l}\text { Action in Diabetes and Vascular disease: } \\ \text { Preterax and Diamicron Modified Release }\end{array} \\ & \begin{array}{l}\text { Controlled Evaluation } \\ \text { MMSE }\end{array}\end{array}$

\section{Introduction}

Diabetes and cognitive dysfunction represent serious health problems in the ageing population, with the prevalence of both conditions projected to increase in most parts of the world [1, 2]. A number of studies have reported that cognitive decline is accelerated in patients with diabetes, independently of common cardiovascular risk factors [3-5] and is associated with poor glycaemic control [6]. In a recent meta-analysis, patients with type 2 diabetes were estimated to have a 1.5-fold greater risk of cognitive dysfunction and 1.6-fold greater risk of dementia compared with people without diabetes [7]. These findings coincide with structural and functional defects demonstrated by various imaging modalities [8], and indicate that cognitive impairment may represent a separate complication alongside retinopathy, nephropathy, neuropathy and cardiovascular disease in patients with type 2 diabetes [8].

Cognitive dysfunction has also been proposed to be a risk factor for cardiovascular disease and mortality in elderly populations [9-11], although this association is probably confounded by the higher prevalence of cardiovascular risk factors such as high BP and type 2 diabetes with increasing age [12]. Among older patients with diabetes, reduced survival has been reported in those with poor cognitive function $[13,14]$. However, previous studies have excluded younger patients, which is a drawback since cognitive function deteriorates at a younger age in patients with type 2 diabetes compared with people without diabetes [8].

Few studies have investigated the effects of BP- and glucose-lowering treatments in patients with both type 2 diabetes and cognitive dysfunction. In the absence of clear cardiovascular benefits, such treatments may be used cautiously or even withheld in these patients due to concerns that lower cerebral blood flow or incident hypoglycaemia may exacerbate cognitive decline and even provoke the onset of dementia [8, 15, 16]. The recent Action in Diabetes and Vascular Disease: Preterax and Diamicron Modified Release Controlled Evaluation (ADVANCE) study, investigated the separate effects of both routine BP lowering and intensive glucose control on vascular outcomes in a broad range of patients with type 2 diabetes $[17,18]$. The purpose of the present analyses was to quantify mortality and cardiovascular risks associated with impaired cognitive function in patients with type 2 diabetes, and to compare the effects of the BP-lowering and intensive glucose-control interventions in those with and without cognitive dysfunction.

\section{Methods}

ADVANCE was a randomised factorial trial designed to investigate the effects of routine BP lowering and intensive glucose control on vascular outcomes in patients with type 2 diabetes. The main results have been reported previously $[17,18]$.

Participants A total of 11,140 patients ( $\geq 55$ years) with a diagnosis of type 2 diabetes (from the age of $\geq 30$ years) and a history of major macro- or microvascular disease or at least one other cardiovascular risk factor, were recruited from 215 centres in 20 countries between June 2001 and 
March 2003 [19]. Patients with a definite indication for long-term insulin therapy at baseline or a definite indication for, or contraindication to, one of the study treatments, were excluded from participating in the study. There were no BP or glucose thresholds for study entry.

Randomised treatments Potentially eligible participants entered a 6 week run-in period, during which they received a fixed combination of the ACE inhibitor, perindopril, and thiazide-like diuretic, indapamide. Patients who tolerated and were compliant with the run-in treatment were subsequently randomised, in a factorial design, to continued treatment with perindopril-indapamide or placebo and to intensive glucose control based on the sulfonylurea, gliclazide MR, targeting an $\mathrm{HbA}_{1 \mathrm{c}}$ of $\leq 6.5 \%$, or to standard glucose control [19]. Median treatment follow-up for the BP-lowering arm of the study was 4.3 years, whereas the glucose-control intervention continued for another 8 months, to be completed after a median follow-up of 5.0 years $[17,18]$.

Definitions Cognitive function was assessed using the Mini Mental State Examination (MMSE) at baseline, at 2 yearly intervals during follow-up, and at study completion. Cognition was defined as 'normal' for MMSE scores of $\geq 28$, as 'mild dysfunction' for scores of $24-27$, and as 'severe dysfunction' for scores $<24$ [20]. Patients with an MMSE score of $<24$ or where there was suspicion of dementia, required referral to an appropriately qualified specialist for diagnosis of dementia according to the criteria in the Diagnostic and Statistical Manual of Mental Disorders, 4th edition (DSM IV), unless a diagnosis of dementia had been made beforehand. The Modification of Diet in Renal Disease equation was used to calculate the estimated GFR [21].

Outcomes The outcomes for the current analysis were major cardiovascular events (non-fatal myocardial infarction, non-fatal stroke, cardiovascular death), all-cause death, cardiovascular death, major coronary events (nonfatal myocardial infarction and death caused by coronary disease), major strokes (non-fatal and fatal stroke), dementia, and any hypoglycaemia (defined as the presence of typical symptoms without other apparent cause or a blood glucose $<2.8 \mathrm{mmol} / \mathrm{l}$ ), and classified as severe (when patients had transient central nervous system dysfunction requiring external assistance) or mild (when self-treated) $[17,19]$.

Statistical analysis The risks of clinical outcomes were analysed by baseline cognitive status using the MMSE categories defined above. Differences in baseline variables between cognitive function subgroups were tested using a
Student's $t$ test, Mann-Whitney test or $\chi^{2}$ test, as appropriate. The risks of events associated with baseline cognitive function were estimated using Cox proportional hazards models, with adjustment for potential confounding baseline covariates: age, sex, treatment allocation, educational status, diabetes duration, systolic BP, history of currently treated hypertension, $\mathrm{HbA}_{1 \mathrm{c}}$, LDL-cholesterol, HDL-cholesterol, BMI, history of macrovascular or microvascular disease, current smoking and current alcohol intake. As the MMSE data were not normally distributed, the risks of clinical outcomes were also examined using baseline MMSE score as a continuous variable in the Cox models.

To determine the extent to which baseline cognitive function modified the effects of randomised treatment, the data were analysed using unadjusted Cox models, according to the principle of intention-to-treat, using 4.3 years of follow-up for the BP-lowering intervention and 5.0 years of follow-up for the glucose-control intervention. Tests of the homogeneity of treatment effects between the cognitive function subgroups were performed by adding an interaction term to the relevant Cox model. All analyses were performed with SAS version 9.1 (SAS Institute, Cary, NC, USA). Data are reported with $95 \%$ CIs and all $p$ values were calculated using two-tailed tests.

\section{Results}

MMSE scores were obtained at baseline in all but eight patients $(<0.1 \%)$, who were excluded from the analyses. In the entire study population the median MMSE score at baseline was 29 (interquartile range 28-30): 8,689 patients (78.1\%) had an MMSE score of $\geq 28,2,231$ patients (20.0\%) had a score of $24-27$, and 212 patients (1.9\%) had a score $<24$, of whom 31 were thought to have dementia. Table 1 shows the baseline characteristics of patients according to level of cognitive function. Patients with a lower MMSE score were older and more often female, and had longer duration of diabetes, fewer years of formal education, and more often diabetic nephropathy or a history of stroke. They also had slightly higher levels of $\mathrm{HbA}_{1 \mathrm{c}}$ and systolic BP, and lower levels of estimated GFR. There were no differences according to assignment to intervention or control groups (data not shown).

Impact of baseline cognitive function on cardiovascular outcomes and mortality During a median follow-up of 5.0 years, a total of 1,147 patients $(10.3 \%)$ developed a major cardiovascular event. In unadjusted analyses, the risks of these events were $52 \%$ and $110 \%$ higher in patients with mild and severe cognitive dysfunction, respectively, 
Table 1 Baseline characteristics of patients

\begin{tabular}{|c|c|c|c|c|}
\hline \multirow[t]{2}{*}{ Characteristics } & \multicolumn{3}{|l|}{ MMSE score } & \multirow[t]{2}{*}{$p$ value for trend } \\
\hline & $28-30(n=8,689)$ & $24-27(n=2,231)$ & $\leq 23(n=212)$ & \\
\hline Age (years) & $65(6.3)$ & $67(6.4)$ & $68(6.2)$ & $<0.001$ \\
\hline Female, $n(\%)$ & $3,538(40.7)$ & $1,078(48.3)$ & $114(53.8)$ & $<0.001$ \\
\hline Age of completion of highest education, years (SD) & $19(7.2)$ & $16(6.8)$ & $12(7.3)$ & $<0.001$ \\
\hline Duration of diabetes (years) & $7.9(6.3)$ & $8.0(6.5)$ & $8.3(7.0)$ & 0.469 \\
\hline History of major cardiovascular disease, $n(\%)$ & $2,761(31.8)$ & $735(32.9)$ & $91(42.9)$ & 0.002 \\
\hline Myocardial infarction, $n(\%)$ & $1,039(12.0)$ & $264(11.8)$ & $30(14.2)$ & 0.607 \\
\hline Stroke, $n(\%)$ & $714(8.2)$ & $260(11.7)$ & $47(22.2)$ & $<0.001$ \\
\hline History of major microvascular disease, $n(\%)$ & $839(9.7)$ & $281(12.6)$ & $35(16.5)$ & $<0.001$ \\
\hline Macroalbuminuria, $n(\%)$ & $280(3.2)$ & $107(4.8)$ & $17(8.0)$ & $<0.001$ \\
\hline Major diabetic eye disease, $n(\%)$ & $588(6.8)$ & $185(8.3)$ & $22(10.4)$ & 0.009 \\
\hline History of hypertension, $n(\%)$ & $5,918(68.1)$ & $1,586(71.1)$ & $145(68.4)$ & 0.024 \\
\hline Past smoker, $n(\%)$ & $3,733(43.0)$ & $865(38.8)$ & $75(35.4)$ & $<0.001$ \\
\hline Current smoker, $n(\%)$ & $1,345(15.5)$ & $309(13.9)$ & $27(12.7)$ & 0.099 \\
\hline Current alcohol use, $n(\%)$ & $2,777(32.0)$ & $572(25.6)$ & $44(20.8)$ & $<0.001$ \\
\hline $\mathrm{HbA}_{1 \mathrm{c}}, \%(\mathrm{SD})$ & $7.5(1.5)$ & $7.6(1.7)$ & $7.7(1.8)$ & $<0.001$ \\
\hline Systolic BP, mmHg (SD) & $144(21.2)$ & $147(22.8)$ & $147(22.5)$ & $<0.001$ \\
\hline Diastolic BP, mmHg (SD) & $81(10.8)$ & $80(11.3)$ & $80(10.7)$ & 0.102 \\
\hline Serum total cholesterol, $\mathrm{mmol} / \mathrm{l}(\mathrm{SD})$ & $5.2(1.2)$ & $5.3(1.2)$ & $5.2(1.1)$ & 0.004 \\
\hline Serum creatinine, $\mu \mathrm{mol} / 1$ & $86.3(24.6)$ & $87.6(28.2)$ & $85.7(23.9)$ & 0.117 \\
\hline 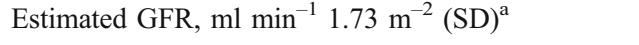 & $83.7(28.6)$ & $77.4(27.8)$ & $75.3(28.2)$ & $<0.001$ \\
\hline Estimated GFR, $<60 \mathrm{ml} \mathrm{min}{ }^{-1} 1.73 \mathrm{~m}^{-2}, n(\%)^{\mathrm{a}}$ & $1,682(19.4)$ & $582(26.2)$ & $64(30.3)$ & $<0.001$ \\
\hline UACR, $\mathrm{mg} / \mathrm{mmol}$ (interquartile range) ${ }^{\mathrm{b}}$ & $1.61(0.79-4.20)$ & $1.93(0.90-5.10)$ & $1.89(0.90-7.23)$ & $<0.001$ \\
\hline BMI $\left(\mathrm{kg} / \mathrm{m}^{2}\right)$ & $28.4(5.2)$ & $28.2(5.0)$ & $28.2(6.3)$ & 0.104 \\
\hline
\end{tabular}

${ }^{a}$ Estimated GFR was calculated using the Modification of Diet in Renal Disease equation [21]

${ }^{\mathrm{b}} \mathrm{UACR}$, urinary albumin to creatinine ratio

compared with those with normal cognitive function (both $p<0.0001)$. These excess risks were attenuated by adjustment for age, sex, treatment allocation, education and a range of cardiovascular risk factors, but remained significant (Table 2). The unadjusted and adjusted risks of major coronary events and of stroke in patients with cognitive dysfunction were elevated to a similar extent to that of the overall risk of major cardiovascular events.

A total of 1,031 patients $(9.3 \%)$ died during follow-up, about half from cardiovascular causes $(n=542,4.9 \%)$. The risks of all-cause and cardiovascular mortality were significantly higher in patients with mild and severe cognitive dysfunction, compared with no dysfunction, in both crude and age-, sex-, treatment allocation- and education-adjusted analyses (all $p<0.001$, Table 2). After further adjustment, the all-cause mortality risks were still $33 \%$ and $50 \%$ higher in patients with mild and severe cognitive dysfunction, respectively, compared with patients with normal cognitive function (both $p<0.03$ ). The increased risk of death was mainly driven by excess cardiovascular mortality in these patient groups.
Impact of baseline cognitive function on hypoglycaemia The risk of severe hypoglycaemia, but not of any hypoglycaemia, was moderately elevated in patients with mild cognitive dysfunction and markedly elevated in patients with severe cognitive dysfunction compared with those with normal cognitive function (Fig. 1). After adjustment for age, sex, treatment allocation and educational status, the risk of severe hypoglycaemia was no longer elevated in patients with mild cognitive dysfunction. However, in patients with severe cognitive dysfunction, even after adjustment for several covariates, the risk of severe hypoglycaemia remained more than twofold higher than in patients with normal cognitive function $(p=0.018)$.

Analyses using MMSE score as a continuous risk factor In age-, sex-, treatment allocation- and education-adjusted analyses, each unit of lower MMSE score at baseline increased the risk of cardiovascular events by $8 \%(95 \% \mathrm{CI}$ $5-11 \%)$, the risk of cardiovascular mortality by $11 \%(95 \%$ CI $8-15 \%)$ and that of all-cause mortality by $9 \%(95 \%$ CI $6-12 \%)($ all $p<0.0001)$. Similarly, for every one unit lower 
Table 2 Results of crude and adjusted analyses of differences in clinical outcomes and adverse events in patients with mild cognitive impairment vs normal cognitive function, and patients with severe cognitive impairment vs normal cognitive function

\begin{tabular}{|c|c|c|c|c|c|c|}
\hline Outcome & Crude analysis & $p$ value & $\begin{array}{l}\text { Adjusted for treatment, } \\
\text { age, sex and education }\end{array}$ & $p$ value & $\begin{array}{l}\text { Adjusted for multiple } \\
\text { covariates }^{\mathrm{a}}\end{array}$ & $p$ value \\
\hline \multicolumn{7}{|c|}{ Mild cognitive dysfunction vs normal cognitive function } \\
\hline Major cardiovascular events & $1.52(1.33-1.74)$ & $<0.0001$ & $1.37(1.20-1.57)$ & $<0.0001$ & $1.27(1.11-1.46)$ & 0.0006 \\
\hline Major strokes & $1.48(1.20-1.81)$ & 0.0002 & $1.34(1.08-1.65)$ & 0.0067 & $1.21(0.97-1.49)$ & 0.0884 \\
\hline Major coronary events & $1.45(1.22-1.74)$ & $<0.0001$ & $1.31(1.09-1.57)$ & 0.0037 & $1.24(1.03-1.49)$ & 0.0246 \\
\hline All heart failure & $1.78(1.46-2.19)$ & $<0.0001$ & $1.51(1.22-1.86)$ & 0.0001 & $1.40(1.13-1.73)$ & 0.002 \\
\hline All-cause mortality & $1.64(1.43-1.88)$ & $<0.0001$ & $1.40(1.22-1.61)$ & $<0.0001$ & $1.33(1.16-1.54)$ & $<0.0001$ \\
\hline Cardiovascular death & $1.79(1.49-2.16)$ & $<0.0001$ & $1.53(1.27-1.85)$ & $<0.0001$ & $1.41(1.16-1.71)$ & 0.0006 \\
\hline New-onset dementia & $4.74(3.12-7.19)$ & $<0.0001$ & $3.71(2.43-5.68)$ & $<0.0001$ & $3.59(2.34-5.50)$ & $<0.0001$ \\
\hline Any hypoglycaemia & $0.97(0.91-1.04)$ & 0.461 & $0.98(0.91-1.05)$ & 0.6056 & $0.98(0.91-1.06)$ & 0.6258 \\
\hline Severe hypoglycaemia & $1.39(1.03-1.89)$ & $<0.0001$ & $1.17(0.86-1.60)$ & 0.3232 & $1.11(0.81-1.53)$ & 0.5102 \\
\hline \multicolumn{7}{|c|}{ Severe cognitive dysfunction vs normal cognitive function } \\
\hline Major cardiovascular events & $2.10(1.52-2.89)$ & $<0.0001$ & $1.80(1.30-2.50)$ & 0.0004 & $1.42(1.01-1.99)$ & 0.0426 \\
\hline Major strokes & $2.01(1.22-3.32)$ & 0.0063 & $1.71(1.03-2.85)$ & 0.0398 & $1.32(0.78-2.25)$ & 0.306 \\
\hline Major coronary events & $1.97(1.27-3.05)$ & 0.0303 & $1.70(1.09-2.64)$ & 0.0200 & $1.33(0.84-2.11)$ & 0.2216 \\
\hline All heart failure & $2.33(1.41-3.85)$ & 0.0010 & $1.80(1.08-3.01)$ & 0.0241 & $1.52(0.91-2.55)$ & 0.1108 \\
\hline All-cause mortality & $2.25(1.62-3.13)$ & $<0.0001$ & $1.83(1.31-2.56)$ & 0.0004 & $1.50(1.06-2.12)$ & 0.0225 \\
\hline Cardiovascular death & $2.76(1.81-4.21)$ & $<0.0001$ & $2.21(1.43-3.40)$ & 0.0003 & $1.56(0.99-2.46)$ & 0.0552 \\
\hline New-onset dementia & $23.4(13.7-40.0)$ & $<0.0001$ & $16.6(9.37-29.6)$ & $<0.0001$ & $16.0(8.93-28.8)$ & $<0.0001$ \\
\hline Any hypoglycaemia & $1.04(0.85-1.27)$ & 0.696 & $1.05(0.86-1.29)$ & 0.6158 & $1.03(0.84-1.26)$ & 0.8059 \\
\hline Severe hypoglycaemia & $3.86(2.24-6.67)$ & $<0.0001$ & $2.86(1.63-5.05)$ & 0.0003 & $2.10(1.14-3.87)$ & 0.018 \\
\hline
\end{tabular}

Values are HRs $(95 \% \mathrm{CIs})$

Normal cognitive function, MMSE score $\geq 28$; mild cognitive impairment, MMSE score 24-27; severe cognitive impairment, MMSE score $\leq 23$ ${ }^{a}$ Adjusted for treatment allocation, age, sex, education, diabetes duration, history of cardiovascular disease, history of microvascular disease, history of hypertension, BMI, systolic BP, HDL-cholesterol, LDL-cholesterol, smoking, alcohol use and $\mathrm{HbA}_{1 \mathrm{c}}$

MMSE score, the risk of severe hypoglycaemia was increased by $10 \%(95 \%$ CI $4-16 \% ; p=0.0010)$. In the multiple-adjusted model, one unit lower MMSE score remained associated with increased risks of cardiovascular events $(6 \%, 95 \%$ CI $3-9 \% ; p<0.0001)$, cardiovascular mortality $(9 \%, 95 \%$ CI $5-13 \% ; p<0.0001)$, all-cause mortality $(8 \%, 95 \%$ CI $5-11 \% ; p<0.0001)$ and severe hypoglycaemia $(7 \%, 95 \%$ CI $1-13 \% ; p=0.0303)$.

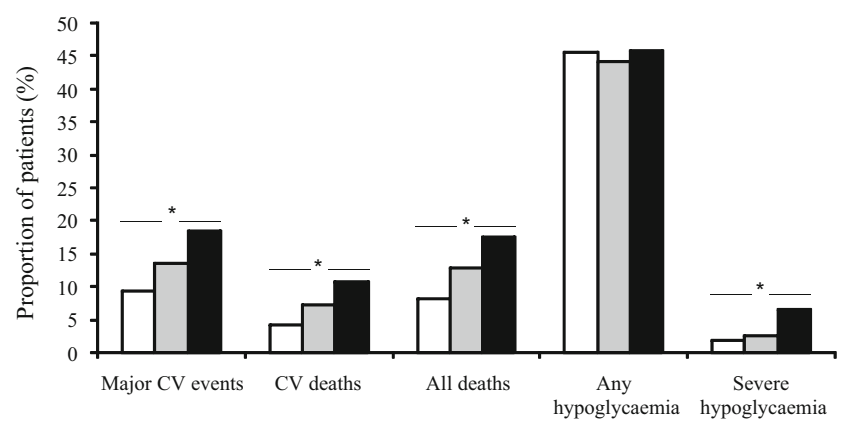

Fig. 1 Proportion of patients with clinical outcomes during follow-up according to cognitive function status at baseline. White bars, normal cognitive function; grey bars, mild cognitive dysfunction; black bars, severe cognitive dysfunction. $\mathrm{CV}$, cardiovascular. ${ }^{*} p$ for trend $<0.0001$
Effects of randomised BP-lowering and glucose-control treatments in cognitive function subgroups There was no indication that baseline cognitive function modulated the effects on major cardiovascular events or cardiovascular death of either the BP-lowering or the intensive glucosecontrol intervention evaluated in ADVANCE (all $p$ values for interaction $\geq 0.3$ ), but there was a suggestion of heterogeneity with respect to the effect of glucose control on all-cause mortality ( $p$ for interaction=0.06) (Fig. 2). Neither intervention modified the risk of cognitive decline or dementia in any of the subgroups (data not shown). As anticipated, intensive glucose control increased the risk of any hypoglycaemia (Fig. 2) but did so to a similar relative extent in the three cognitive function subgroups ( $p$ for interaction $=0.69$ ).

\section{Discussion}

In these analyses of the ADVANCE study, patients with type 2 diabetes and mildly impaired cognitive function, as assessed by the MMSE were at increased risk of cardio- 
Fig. 2 Effects of randomised interventions on major clinical outcomes. The centres of the diamonds represent the estimates and their widths, with the $95 \%$ CIs for overall treatment effect. Solid boxes represent estimates of treatment effect in subgroups: the centres of the boxes are placed at the estimates of effect, the areas of boxes are proportional to the number of events, and horizontal lines represent the corresponding $95 \%$ CIs. The vertical dotted line represents the point estimate for overall effect. The interaction $p$ value tested the consistency of treatment effect in subgroups. a Effect of intensive vs standard glucose control on major cardiovascular events, all-cause mortality, cardiovascular death and hypoglycaemia in subgroups of participants defined by cognitive function at baseline. b Effect of routine perindoprilindapamide vs placebo BPlowering treatment on major cardiovascular events, all-cause mortality and cardiovascular death in subgroups of participants defined by cognitive function at baseline a

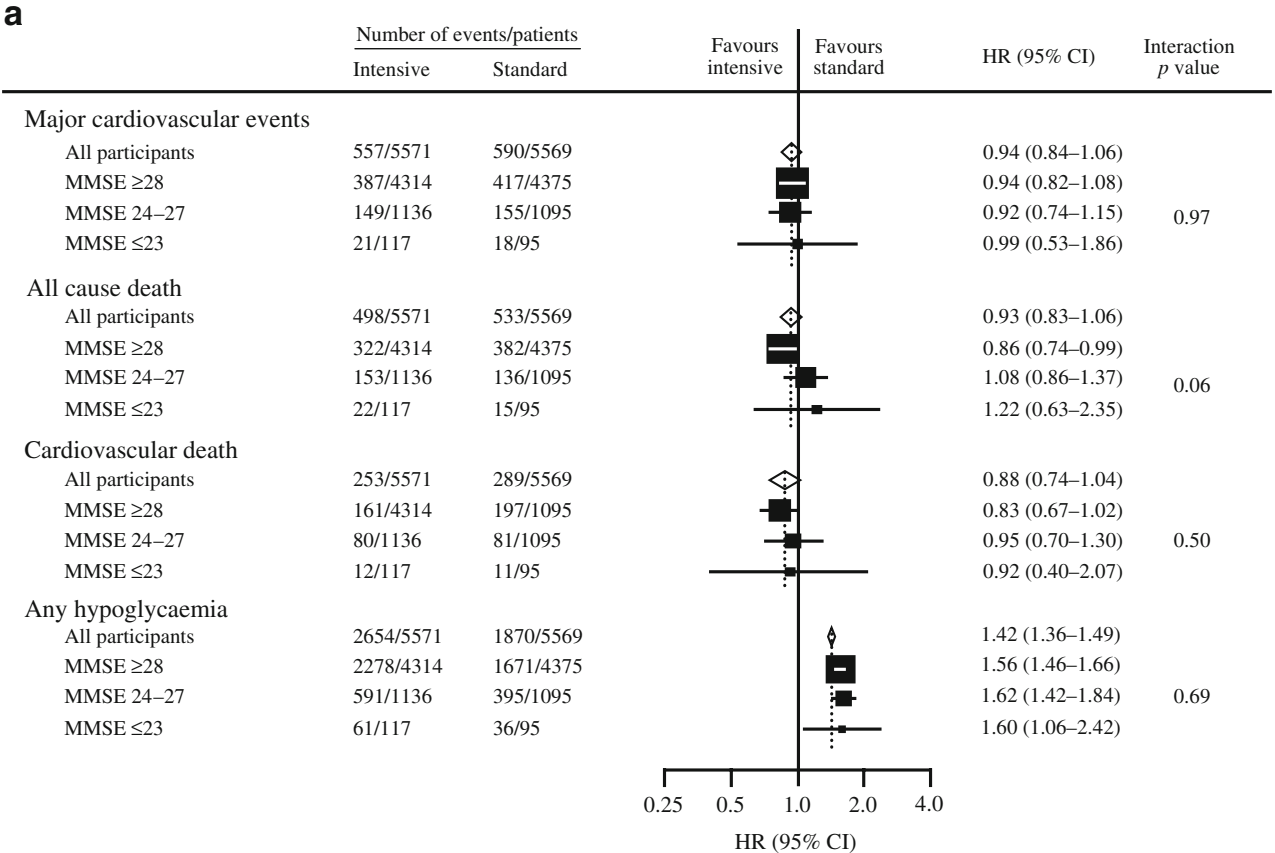

b

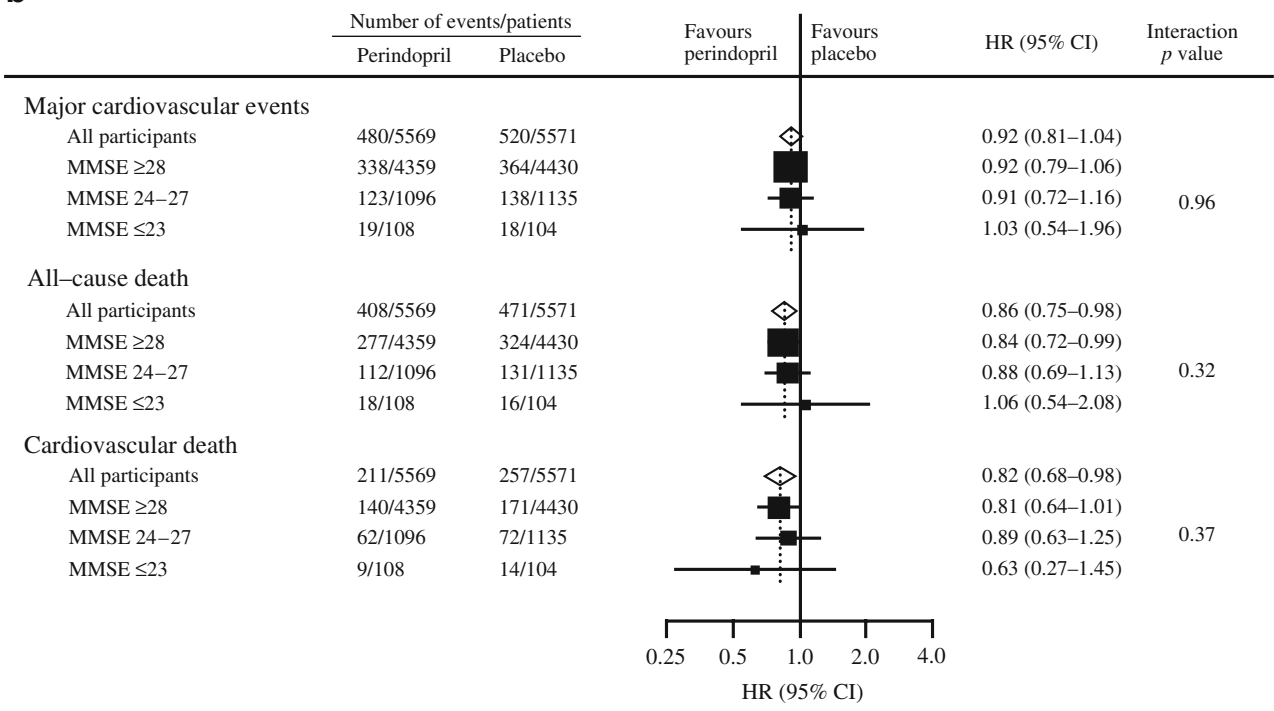

vascular events and death. Patients with more severe cognitive dysfunction were at even greater risk of such events and were also at increased risk of severe hypoglycaemia. For all these outcomes, there was an inverse and continuous correlation with the MMSE score, in that the lower the MMSE score the higher the event risk. There was no evidence to suggest that cognitive dysfunction modified the response to BP-lowering or glucose-control treatments in the management of cardiovascular risk. Thus, patients with cognitive dysfunction appeared to benefit to the same extent as patients with normal cognitive function from interventions that reduce the risks of cardiovascular outcomes. As previously reported, in this group of patients with type 2 diabetes neither BP lowering nor intensive glucose control had any effect on the development of cognitive impairment and dementia $[17,18]$.

Our findings of increased mortality and cardiovascular disease risks associated with baseline cognitive impairment are in agreement with observations in the general elderly population [10,11, 13, 22], and in older patients with diabetes [14]. Adjusting for level of education did not materially alter these risks (data not shown, but available on request), making it unlikely that our results were merely an extension of a low level of intelligence, which by itself carries an increased cardiovascular risk [23, 24]. Much of the increased risk may be explained by the higher prevalence of cardiovascular risk factors among patients with cognitive dysfunction. However, although attenuated, 
the association between impaired cognitive function and major clinical outcomes in our study remained statistically significant even after controlling for these risk factors. This may be caused in part by incomplete adjustment for and consequent overestimation of covariates measured with substantial error, and in part to covariates that were unmeasured or otherwise unknown, such as subclinical vascular disease or poor compliance with pharmacological and non-pharmacological treatments [25].

A potential biological factor that could explain the link between cognitive dysfunction and cardiovascular events concerns chronic hyperinsulinaemia or insulin resistance. Chronic hyperinsulinaemia has been associated with both cognitive dysfunction [26] and increased risk of cardiovascular death [27]. There has also been an association between low beta cell function, a determinant of type 2 diabetes and thus of chronic hyperinsulinaemia later in life, and Alzheimer's disease [28]. Insulin resistance, a wellknown risk factor for atherosclerosis [29], has been associated with vascular dementia [28]. Although once considered insulin-insensitive, the brain is now thought to depend on intact insulin signalling for several aspects of its function, including memory formation. Chronic hyperinsulinaemia and peripheral insulin resistance may impair insulin signalling by reducing cerebral insulin uptake [30] and insulin action [31], respectively. They may also impair memory function and precede Alzheimer's disease by increasing the levels of inflammatory factors and $\beta$ amyloid in the brain [32].

Patients with severe cognitive dysfunction were at twofold higher risk of severe hypoglycaemia than patients with normal cognitive function. Thus while both patients and physicians share concern that severe hypoglycaemia may be implicated in the aetiology of cognitive impairment [8], they should also be aware of a possible causal relationship in the opposite direction. The higher risk of severe hypoglycaemia was independent of recognised risk factors such as old age, low $\mathrm{HbA}_{1 \mathrm{c}}$, long duration of diabetes and cardiovascular risk factors. However, it is conceivable that incomplete adherence to or inappropriate use of the glucose-control regimen, which could not be assessed with certainty, might have played a role. Management of diabetes is complex and heavily dependent on active involvement of patients with respect to drug compliance, glucose testing, meal planning and insulin dose titration. This is a demanding process that could cause greater difficulties for patients with severe cognitive impairment. Nevertheless, our analyses did not reveal increased relative risks of hypoglycaemia associated with intensive glucose-control intervention in patients with mild or severe cognitive dysfunction. However, information on mild hypoglycaemia was only collected on the basis of selfreporting, which may be less reliable in the cognitively impaired. Furthermore, there were too few severe hypoglycaemic episodes overall to have adequate power for this subgroup analysis.

There are limited data on the efficacy of risk-factor management in people with impaired cognitive function. Among elderly people with hypertension, BP-lowering treatment was recently reported to reduce the risk of stroke to the same extent as in patients with mildly impaired or normal cognitive function [12]. Our data support these findings in that the relative benefits of BP-lowering treatment and risks of intensive glucose control in patients with type 2 diabetes were largely independent of the level of cognitive function. The greater baseline risk of different outcomes in patients with cognitive dysfunction may translate these similar relative treatments effects into both greater absolute benefits and greater absolute risks (for example severe hypoglycaemia). In this respect, there was some suggestion that cognitive function modified the effect of glucose lowering on survival, in that patients with lower MMSE scores benefited less than those with higher scores. Although this finding was probably caused by chance, it supports the importance of balancing potential benefits and risks for each patient when making treatment decisions. In any case, there seems little justification in denying patients risk-modifying treatment solely on the basis of cognitive impairment.

Some limitations of this study merit consideration. First, the MMSE was originally designed as a screening test for dementia and not especially for the assessment of milder degrees of cognitive dysfunction [33]. However, this limitation may be less relevant for the use of MMSE in cohort or epidemiological studies. Second, at baseline most patients had maximal MMSE scores (i.e. normal cognitive function) with only a small proportion $(<2 \%)$ demonstrating severe impairment of cognitive function. Thus there was limited statistical power to analyse risks associated with severe cognitive dysfunction. Yet when analysing risks of clinical outcomes by using MMSE as a continuous variable, similar results were yielded, substantiating the validity of our results, at least with respect to mild cognitive dysfunction. As indicated previously, there was insufficient power to analyse the risk of severe hypoglycaemia by randomised glucose-control assignment in cognitive function subgroups.

In conclusion, our data show that cognitive dysfunction further increases the already greater risk of cardiovascular events and death, but does not adversely modify the response to BP- or glucose-lowering treatment in patients with type 2 diabetes. This is clinically relevant, as cognitive decline is common among patients with type 2 diabetes and may influence management [8]. Our data do not support commonly held views $[8,15,16]$ suggesting general restraints with regard to cardiovascular risk management 
in patients with cognitive impairment. Therefore, rather than denying patients with type 2 diabetes risk-modifying treatment on the basis of cognitive dysfunction, such patients deserve a similar careful balancing of all potential risks and benefits associated with treatment to that of patients with intact cognitive function. Future guidelines may need to address this growing patient group in their directives for clinical care.

Acknowledgements ADVANCE was funded by grants from Servier and the National Health and Medical Research Council of Australia. The sponsors had no role in the design of the study, data collection, data analysis, data interpretation and the writing of the manuscript. Study data were not made available to the sponsors. The Management Committee, whose membership did not include any sponsor representatives, had final responsibility for the decision to submit for publication. The first four authors had full access to the data of the study and take responsibility for the accuracy of the analysis.

Duality of interest J. Chalmers and S. MacMahon hold research grants from Servier as principal investigators for ADVANCE. B. E. de Galan, S. Zoungas, J. Chalmers, M. Cooper, D. E. Grobbee, P. Hamet, S. MacMahon, G. Mancia, B. Neal, C. Y. Pan, A. Patel, N. Poulter, F. Travert and M. Woodward have received lecturing fees from Servier. C. Anderson, C. Dufouil, A. Pillai, M. Hackett, S. R. Heller and L. Lisheng declare that there is no duality of interest associated with this manuscript.

\section{References}

1. Ferri CP, Prince M, Brayne C et al (2005) Global prevalence of dementia: a Delphi consensus study. Lancet 366:2112-2117

2. Wild S, Roglic G, Green A, Sicree R, King H (2004) Global prevalence of diabetes: estimates for the year 2000 and projections for 2030. Diabetes Care 27:1047-1053 (see comment)

3. Cukierman-Yaffe T, Gerstein HC, Anderson C et al (2009) Glucose intolerance and diabetes as risk factors for cognitive impairment in people at high cardiovascular risk: results from the ONTARGET/TRANSCEND research programme. Diabetes Res Clin Pract 83:387-393

4. Fontbonne A, Berr C, Ducimetiere P, Alperovitch A (2001) Changes in cognitive abilities over a 4-year period are unfavorably affected in elderly diabetic subjects: results of the Epidemiology of Vascular Aging Study. Diabetes Care 24:366-370

5. Gregg EW, Yaffe K, Cauley JA et al (2000) Is diabetes associated with cognitive impairment and cognitive decline among older women? Study of Osteoporotic Fractures Research Group. Arch Intern Med 160:174-180 (see comment)

6. Cukierman-Yaffe T, Gerstein HC, Williamson JD et al (2009) Relationship between baseline glycemic control and cognitive function in individuals with type 2 diabetes and other cardiovascular risk factors: the action to control cardiovascular risk in diabetes-memory in diabetes (ACCORD-MIND) trial. Diabetes Care 32:221-226

7. Cukierman T, Gerstein HC, Williamson JD (2005) Cognitive decline and dementia in diabetes - systematic overview of prospective observational studies. Diabetologia 48:2460-2469

8. Kodl CT, Seaquist ER (2008) Cognitive dysfunction and diabetes mellitus. Endocr Rev 29:494-511

9. Breteler MM, Claus JJ, Grobbee DE, Hofman A (1994) Cardiovascular disease and distribution of cognitive function in elderly people: the Rotterdam Study. BMJ 308:1604-1608 (see comment)

10. Ferrucci L, Guralnik JM, Salive ME et al (1996) Cognitive impairment and risk of stroke in the older population. J Am Geriatr Soc 44:237-241 (see comment)

11. Gale CR, Martyn CN, Cooper C (1996) Cognitive impairment and mortality in a cohort of elderly people. BMJ 312:608-611

12. Skoog I, Lithell H, Hansson L et al (2005) Effect of baseline cognitive function and antihypertensive treatment on cognitive and cardiovascular outcomes: Study on COgnition and Prognosis in the Elderly (SCOPE). Am J Hypertens 18:1052-1059

13. Feil D, Marmon T, Unutzer J (2003) Cognitive impairment, chronic medical illness, and risk of mortality in an elderly cohort. Am J Geriatr Psychiatry 11:551-560

14. McGuire LC, Ford ES, Ajani UA (2006) The impact of cognitive functioning on mortality and the development of functional disability in older adults with diabetes: the second longitudinal study on aging. BMC Geriatr 6:8

15. Nilsson SE, Read S, Berg S, Johansson B, Melander A, Lindblad U (2007) Low systolic blood pressure is associated with impaired cognitive function in the oldest old: longitudinal observations in a population-based sample 80 years and older. Aging Clin Exp Res 19:41-47

16. Zhu L, Viitanen M, Guo Z, Winblad B, Fratiglioni L (1998) Blood pressure reduction, cardiovascular diseases, and cognitive decline in the mini-mental state examination in a community population of normal very old people: a three-year follow-up. J Clin Epidemiol 51:385-391

17. Patel A, MacMahon S, Chalmers J et al (2008) Intensive blood glucose control and vascular outcomes in patients with type 2 diabetes. N Engl J Med 358:2560-2572

18. Patel A, MacMahon S, Chalmers J et al (2007) Effects of a fixed combination of perindopril and indapamide on macrovascular and microvascular outcomes in patients with type 2 diabetes mellitus (the ADVANCE trial): a randomised controlled trial. Lancet 370:829-840

19. ADVANCE Management Committee (2001) Study rationale and design of ADVANCE: action in diabetes and vascular diseasepreterax and diamicron MR controlled evaluation. Diabetologia 44:1118-1120

20. Crum RM, Anthony JC, Bassett SS, Folstein MF (1993) Population-based norms for the Mini-Mental State Examination by age and educational level. JAMA 269:2386-2391 (see comment)

21. Sarnak MJ, Levey AS, Schoolwerth AC et al (2003) Kidney disease as a risk factor for development of cardiovascular disease: a statement from the American Heart Association Councils on Kidney in Cardiovascular Disease, High Blood Pressure Research, Clinical Cardiology, and Epidemiology and Prevention. Circulation 108:2154-2169

22. Elkins JS, Knopman DS, Yaffe K, Johnston SC (2005) Cognitive function predicts first-time stroke and heart disease. Neurology 64:1750-1755

23. Batty GD, Mortensen EL, Nybo Andersen AM, Osler M (2005) Childhood intelligence in relation to adult coronary heart disease and stroke risk: evidence from a Danish birth cohort study. Paediatr Perinat Epidemiol 19:452-459

24. Batty GD, Wennerstad KM, Smith GD et al (2009) IQ in early adulthood and mortality by middle age: cohort study of 1 million Swedish men. Epidemiology 20:100-109

25. Perlmuter LC, Hakami MK, Hodgson-Harrington C et al (1984) Decreased cognitive function in aging non-insulin-dependent diabetic patients. Am J Med 77:1043-1048

26. Stolk RP, Breteler MM, Ott A et al (1997) Insulin and cognitive function in an elderly population. The Rotterdam Study. Diabetes Care 20:792-795 
27. Hu G, Qiao Q, Tuomilehto J et al (2004) Plasma insulin and cardiovascular mortality in non-diabetic European men and women: a meta-analysis of data from eleven prospective studies. Diabetologia 47:1245-1256

28. Rönnemaa E, Zethelius B, Sundelof J et al (2009) Glucose metabolism and the risk of Alzheimer's disease and dementia: a population-based 12 year follow-up study in 71-year-old men. Diabetologia 52:1504-1510

29. Howard G, O'Leary DH, Zaccaro D et al (1996) Insulin sensitivity and atherosclerosis. The Insulin Resistance Atherosclerosis Study (IRAS) Investigators. Circulation 93:1809-1817

30. Baura GD, Foster DM, Kaiyala K, Porte D Jr, Kahn SE, Schwartz MW (1996) Insulin transport from plasma into the central nervous system is inhibited by dexamethasone in dogs. Diabetes 45:86-90

31. Anthony K, Reed LJ, Dunn JT et al (2006) Attenuation of insulin-evoked responses in brain networks controlling appetite and reward in insulin resistance: the cerebral basis for impaired control of food intake in metabolic syndrome? Diabetes 55: 2986-2992

32. Fishel MA, Watson GS, Montine TJ et al (2005) Hyperinsulinemia provokes synchronous increases in central inflammation and betaamyloid in normal adults. Arch Neurol 62:1539-1544

33. Folstein MF, Folstein SE, McHugh PR (1975) Mini-mental state. A practical method for grading the cognitive state of patients for the clinician. J Psychiatr Res 12:189-198 